\title{
Human apurinic/apyrimidinic endonuclease 1 is modified by poly(ADP-ribose) polymerase 1 via the DNA structure- controlled mechanism
}

\author{
Nina Moor \\ Institute of Chemical \\ Biology and Fundamental \\ Medicine (ICBFM), \\ Siberian Branch of the \\ Russian Academy of \\ Sciences \\ Novosibirsk, Russia
}

\author{
Inna Vasil'eva \\ Institute of Chemical \\ Biology and Fundamental \\ Medicine (ICBFM), \\ Siberian Branch of the \\ Russian Academy of \\ Sciences \\ Novosibirsk, Russia
}

\author{
Nikita Kuznetsov \\ Institute of Chemical \\ Biology and Fundamental \\ Medicine (ICBFM), \\ Siberian Branch of the \\ Russian Academy of \\ Sciences \\ Novosibirsk, Russia
}

\author{
Olga Lavrik \\ Institute of Chemical \\ Biology and Fundamental \\ Medicine (ICBFM), \\ Siberian Branch of the \\ Russian Academy of \\ Sciences \\ Novosibirsk, Russia
}

\begin{abstract}
Apurinic/apyrimidinic endonuclease 1 (APE1) is an essential multifunctional protein in mammals involved in base excision DNA repair (BER) and other processes. Poly(ADP-ribose) polymerase 1 (PARP1) modifies itself and target proteins with poly(ADP-ribose), contributing to regulation of many processes. To understand molecular basis of cooperation between APE1 and PARP1 in BER, we examined poly(ADP-ribose)-binding activity and ADP-ribosylation of human APE1 in comparison with known targets of PARP1, using full-length, N-terminally truncated and catalytically inactive APE1 forms. The protein binds preferentially large ADP-ribose polymers, being similar to DNA polymerase $\beta$ (Pol $\beta$ ) but contrasting with the scaffold XRCC1 protein. The interaction with poly(ADP-ribose) involves the universally conserved portion and the eukaryote-specific extension of APE1. The ADP-ribosylation of APE1 depends on the structure of PARP1-activating DNA, contrasting APE1 with Pol $\beta$ and XRCC1. Relative levels of APE1 modification in the presence of different DNAs were found to correlate with affinities of the DNAs for APE1 and substrate activities in the enzymatic incision, suggesting the ADP-ribosylation to occur within the DNAmediated complex. This conclusion was confirmed by importance of the length of DNA region 3' to the AP site for the modification. Deletion of the N-terminal extension of APE1 produced no significant influence on the ADP-ribosylation and hydrolytic stability of the modified protein, suggesting localization of target residues in the catalytic core. The most efficient ADP-ribosylation of the inactive APE1 reduced the level of PARP1 automodification, suggesting role of APE1 in modulating PARP1 activity. Our data provide new insights into mechanisms of protein targeting for ADP-ribosylation.
\end{abstract}

Keywords - apurinic/apyrimidinic endonuclease 1, poly(ADPribose) polymerase 1, protein ADP-ribosylation, base excision repair, posttranslational modification

\section{Introduction}

APE1 is an essential protein in mammals with multiple functions in BER, regulation of gene expression and RNA metabolism [1]. BER is a molecular pathway devoted to correction of apurinic/apyrimidinic (AP) sites, modified bases and single-strand breaks (SSBs) [2, 3]. The major enzymatic function of APE1 in BER is incision of AP sites. In addition, APE1 can remove terminal blocking groups of BER DNA intermediates. The multiple activities of APE1 are modulated by protein-protein interactions and post-translational modifications $[4,5]$. PARP1 is among the proteins modulating the APE1 activities in BER $[6,7]$. This abundant nuclear protein acts as a rapid DNA damage sensor activated by binding to SSBs or double-strand breaks [2]. The subsequent PARP1 automodification with poly(ADP-ribose) (PAR) is a key mechanism for coordination of BER, via the PAR-dependent interaction with other BER proteins, primarily with a scaffold $\mathrm{X}$-ray repair cross-complementing protein 1 (XRCC1), which in turn interacts with multiple BER enzymes. PARP1-catalysed modification of proteins modulates their properties and functions [2, 5]. A regulatory role of PARP1 in BER is also based on direct interactions with enzymes and competition for the interaction with DNA intermediates [6-8]. We have shown direct interaction of APE1 with PARP1 and its DNA-dependent modulation [8]. To further extend our knowledge of molecular mechanisms underlying the APE1-PARP1 interaction, we examined the non-covalent binding of APE1 to PAR and PARP1-catalysed ADP-ribosylation of APE1. The study was performed with the full-length human APE1, its N-terminally truncated forms deprived of the entire eukaryote-specific extension (APE1N $\Delta 61)$ or its disordered fragment (APE1N $\Delta 35)$ and an inactive APE1 D210N mutant to localize the structural regions involved in the non-covalent and covalent interaction with PAR. The ADP-ribosylation of APE1 was explored in detail, using different synthetic DNA intermediates of BER and their structural variants.

\section{Results and Discussion}

\section{Characterization of PAR-binding activity of APE1}

Proteins identified as targets of poly(ADP-ribosyl)ation interact non-covalently with PAR [9]. Therefore we first explored the non-covalent interaction of APE1 with PAR in comparison with Pol $\beta$ and XRCC1, using partially fractionated polymer. APE1 and Pol $\beta$ bind preferentially large linear and branched polymers ( $>20$-mers) with similar parameters, in contrast to high-affinity interaction of XRCC1 with small ADPribose polymers. Using the full-length and truncated forms of APE1 it was shown that both the N-terminal eukaryote-specific extension and the C-terminal universally conserved catalytic portion of APE1 are involved in PAR binding, with the first of them contributing for the most part to the interaction with linear polymers [10]. The positively charged part of the extension conserved in mammals is critically important for the interaction with small polymers (6-10-mers). Overlapping of PAR- and DNA-binding domains in both APE1 and Pol $\beta$ was shown in the competitive binding experiments performed in the presence of DNA intermediates.

\section{Covalent ADP-ribosylation of APE1 catalysed by PARP1}

The ADP-ribosylation of APE1 was explored in parallel with known targets of PARP1, Pol $\beta$ and XRCC1, using various DNAs for PARP1 activation. To exclude impact of DNA incision at AP site, the experiments were performed with the 
wild-type APE1 and catalytically inactive D210N mutant in the presence of calcium ions as PARP1 cofactor. The ADPribosylation of Pol $\beta$ and $\mathrm{XRCC} 1$ is independent on the presence and type of damage (intact/incised AP site or gap) in the DNA duplex. The most efficient modification of APE1 was detected in the presence of 32-mer DNA duplex containing a medially located synthetic AP site and a purine base in the opposite position (F/Pur-d32). The relative levels of APE1 ADPribosylation in the presence of DNA variants correlate with their affinities for APE1, indicating that the modification is controlled by the strength of APE1-DNA interaction and occurs within the ternary complex of proteins with DNA. The alternative PARmediated mode of APE1 interaction with PARP1 is excluded by the fact that deletion of the N-terminal extension of APE1 contributing to the interaction with PAR revealed no negative influence on the ADP-ribosylation efficiency. The primary role of DNA in proper positioning of APE1 within the ternary complex is further evidenced by results obtained in experiments with sequence variants of F/A-d32 DNA. The effects produced by the sequence variations on the initial rates of the APE1catalysed AP-DNA incision in multiple-turnover conditions and on the ADP-ribosylation efficiency of APE1 (both wild-type and truncated/mutant forms) well correlate with each other. These results indicate that, in addition to the affinity of APE1 for DNA, the conformational dynamics of APE1-DNA contacts mediating substrate binding and product release determines the efficiency of PARP1-catalysed modification of APE1, thus suggesting localization of the acceptor residue(s) in the catalytic core of APE1.

The experiments performed with truncated and elongated variants of F/A-d32 DNA have shown that the efficiency of PARP1-catalysed APE1 modification is determined by the length of base-paired region 3' to the AP site. PARP1 is activated predominantly via interaction with the blunt ends of DNA duplex [11]. In the X-ray structure of its complex with DNA duplex, each end of the duplex is independently bound to the protein via interactions of Zn1 and WGR domains with 3' and 5' terminated DNA strands respectively [12]. Therefore, the catalytic domains of PARP1 bound to the DNA ends in the proposed ternary complex are oriented to opposite directions relative to the AP site and as a consequence relative to the bound APE1 (Fig. 1). Our results show that PARP1 bound to the 3' end of AP site-containing strand is capable of catalysing the modification of APE1. The optimal length between the binding sites of APE1 and PARP1 in the DNA was estimated being about 20 base pairs.

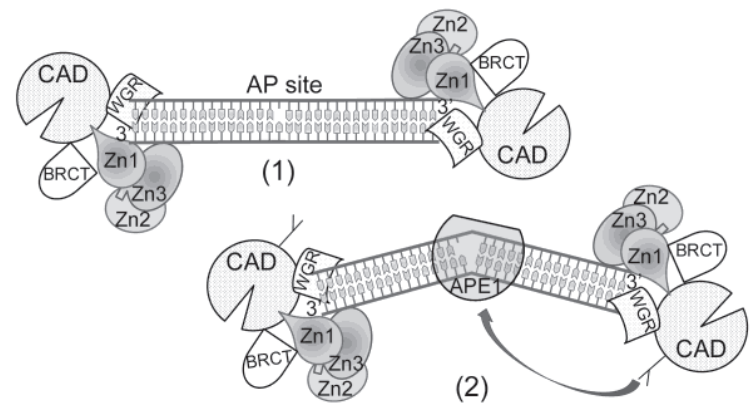

Fig. 1. A mechanism of PARP1-catalysed ADP-ribosylation of APE1 controlled by the DNA structure. State (1) shows PARP1 binding to AP site-containing DNA. PARP1 contains three Zn-finger domains, WGR, BRCT and the catalytic (CAD) domains positioned in accordance with the PARP1-DNA structure [12]; the 3' and 5' ends of each DNA strand are bound to the Zn1 and WGR domains respectively. Specific interaction of APE1 with AP site (state 2) results in kinking the DNA. PARP1 bound 3' to the AP site at a distance of about two helical turns is shown to be capable of catalysing the modification of APE1.
Our studies provide evidence that diverse mechanisms may be responsible for the functional cooperation between APE1 and PARP1. The ADP-ribosylation of APE1 and direct interaction between APE1 and PARP1 modulated by the structure of DNA intermediates are evidently related to assistance between the proteins during DNA repair. The unusual mechanism of protein ADP-ribosylation provides new insights into mechanisms of protein targeting for the post-translational modification with ADP-ribose. DNA acting as the activator of PARP1 can bring the catalytic domain of PARP1 in close proximity to the acceptor protein and thereby play a direct role in the substrate targeting.

\section{ACKNOWLEDGMENT}

This work was supported by the Russian Science Foundation 19-14-00107. Protein purification was supported by grant of Russian Fund for Basic Research 17-04-00925.

\section{REFERENCES}

[1] G. Antoniali, M. C. Malfatti, and G. Tell, Unveiling the non-repair face of the Base Excision Repair pathway in RNA processing: A missing link between DNA repair and gene expression? DNA Repair, 56 (2017) 6574.

[2] R. Abbotts, and D. M. Wilson 3rd, Coordination of DNA single strand break repair, Free Radic. Biol. Med., 107 (2017) 228-244.

[3] A. M. Whitaker, M. A. Schaich, M. R. Smith, T. S. Flynn, and B. D. Freudenthal, Base excision repair of oxidative DNA damage: from mechanism to disease, Front. Biosci. (Landmark Ed.), 22 (2017) 14931522 .

[4] S. Thakur, M. Dhiman, G. Tell, and A. K. Mantha, A review on proteinprotein interaction network of APE1/Ref-1 and its associated biological functions, Cell Biochem. Funct., 33 (2015) 101-112.

[5] K. L. Limpose, A. H. Corbett, and P. W. Doetsch, BERing the burden of damage: pathway crosstalk and posttranslational modification of base excision repair proteins regulate DNA damage management, DNA Repair, 56 (2017) 51-64.

[6] M. V. Sukhanova, S. N. Khodyreva, N. A. Lebedeva, R. Prasad, S. H. Wilson, and O. I. Lavrik, Human base excision repair enzymes apurinic/apyrimidinic endonuclease1 (APE1), DNA polymerase beta and poly(ADP-ribose) polymerase 1: interplay between strand-displacement DNA synthesis and proofreading exonuclease activity, Nucleic Acids Res., 33 (2005) 1222-1229.

[7] S. N. Khodyreva, R. Prasad, E. S. Ilina, M. V. Sukhanova, M. M. Kutuzov, Y. Liu, E. W. Hou, S. H. Wilson, and O. I. Lavrik, Apurinic/apyrimidinic (AP) site recognition by the 5'-dRP/AP lyase in poly(ADP-ribose) polymerase-1 (PARP-1), Proc. Natl. Acad. Sci. U.S.A., 107 (2010) 22090-22095.

[8] N. A. Moor, I. A. Vasil'eva, R. O. Anarbaev, A. A. Antson, and O. I. Lavrik, Quantitative characterization of protein-protein complexes involved in base excision DNA repair, Nucleic Acids Res., 43 (2015) 6009-6022.

[9] J. P. Gagné, M. Isabelle, K. S. Lo, S. Bourassa, M. J. Hendzel, V. L. Dawson, T. M. Dawson, and G. G. Poirier, Proteome-wide identification of poly(ADP-ribose) binding proteins and poly(ADP-ribose)-associated protein complexes, Nucleic Acids Res., 36 (2008) 6959-6976.

[10] N. A. Moor, I. A. Vasil'eva, N. A. Kuznetsov, and O. I. Lavrik, Human apurinic/apyrimidinic endonuclease 1 is modified in vitro by poly(ADPribose) polymerase 1 under control of the structure of damaged DNA, Biochimie, 168 (2020) 144-155.

[11] W. Lilyestrom, M. J. van der Woerd, N. Clark, and K. Luger, Structural and biophysical studies of human PARP-1 in complex with damaged DNA, J. Mol. Biol., 395 (2010) 983-994.

[12] M. F. Langelier, J. L. Planck, S. Roy, and J. M. Pascal, Structural basis for DNA damage-dependent poly(ADP-ribosyl)ation by human PARP-1, Science, 336 (2012) 728-732. 